\title{
STUDY ON PERFORMANCE ANALYSIS OF POLARIZATION RECONFIGURABLE ANTENNA USING METASURFACE
}

\author{
Jnana N J \\ Communication Systems, Electronics and Communication Engineering, \\ TKM College of Engineering, Kollam, India
}

\author{
Abin R \\ Junior Telecom Officer, BSNL, India
}

\begin{abstract}
Performance of a polarization reconfigurable antenna using Metasurface is studied. Polarization of a normal patch antenna can be made reconfigurable by rotating the MS, placed along with the patch antenna. The MS of circular shape is placed above the circular patch antenna with circular substrate. By rotating the MS mechanically with respect to the patch antenna, the polarization of the antenna is reconfigured to Linear polarization (LP) and circular polarization $(C P)$. The radiation pattern, reflection coefficient and axial ratio (AR) are analyzed. The gain and bandwidth of the patch antenna is improved by using a MS. Results show that the PRMS antenna in circular polarization achieves an operating bandwidth of $2.4-3$ $\mathrm{GHz}$
\end{abstract}

Key words: Polarization Reconfigurable Antenna, Metasurface (MS), Patch Antenna

Cite this Article: Jnana N J and Abin R, Study on Performance Analysis of Polarization Reconfigurable Antenna Using Metasurface. International Journal of Electronics and Communication Engineering and Technology, 10(1), 2019, pp. 17-24. http://iaeme.com/Home/issue/IJECET?Volume=10\&Issue $=1$

\section{INTRODUCTION}

Postures of mobile Communication devices in use are often dynamically changing which creates difficulties to have good polarization matching for antennas with only single polarization, it is desirable to have antennas to be able to work in different polarizations such as linear polarization (LP) and circular polarization (CP) [1]. A polarization reconfigurable antenna, can offer various polarizations at the same operating frequency (ie CP and LP), has received considerable attention because it has the potential to improve the performances of wireless communication systems [2]. A Polarization reconfigurable antenna structure can be achieved by placing a planar structured metasurface along the base antenna the polarization can be reconfigured [7]. 
In this paper a mechanically reconfigurable antenna is presented. The antenna is reconfigured to various polarizations by using a metasurface (MS) [1]. MS is a two dimensional equivalent of metamaterial. Metamaterials are typically engineered by arranging a set of small scatterers in a regular array throughout a region of space. By placing a MS atop the patch antenna makes a noticeable change in the characteristics of the patch antenna [7]. The patch antenna is used here due to their low profile, light weight and easy fabrication [9]. By rotating the metasurface the polarization characteristics of the antenna varies from Linear polarization (LP) to Circular polarization (CP).

The Patch antenna along with the MS is called PRMS antenna [1]. The performance of the PRMS antenna having a square shaped truncated unit cells is analyzed in this paper.

The structure of this paper is as follows. Section 2 describes about the design of PRMS antenna. The modeling of the antenna is explained in section 3. Section 4 highlights the simulation and measurement results. Conclusion is discussed in section 5.

\section{DESIGN OF PRMS ANTENNA}

The PRMS antenna consists of a metasurface and a source antenna. The source antenna used here is a single feed circular patch antenna [11]. Metasurface consist of a $4 \times 4$ square unit cells. The two edges of the unit cell is truncated with a small square unit cell. The MS is very simple in structure and it has only few parameters. The MS along with the truncated square cell is shown in Fig 1. The MS contains a single sided substrate and the patch antenna consists of a double sided substrate. Both the patch antenna and MS are designed to have a circular shape for easy mechanical operation. The polarization reconfigurability of the antenna is obtained by rotating the MS with respect to the patch antenna. The MS is rotated with respect to $\mathrm{Y}$ axis. The rotation is expressed in terms of rotation angle $\theta_{R}$. When the MS is at $0^{0}$ the antenna shows linearly polarized (LP) characteristics. When the MS is at $45^{\circ}$ the antenna shows Right hand Circularly Polarized (RHCP) characteristics. When the MS is at $90^{\circ}$ the antenna shows a LP characteristics. When the MS is at $135^{\circ}$ the antenna shows Left Hand Circularly Polarized characteristics.

The substrate portion of the MS is placed on the patch antenna. The SMA connector is used to feed to the feed line through the ground plane and the substrate material of the patch antenna. The PRMS antenna together with the coaxial feed is studied using CST EM simulation tool. The FR-4 (lossy) substrate with a thickness (h) of $1.5 \mathrm{~mm}$ and a dielectric constant of $\varepsilon_{r}=4.3$ is used.

\section{THE MODELLING OF THE PRMS ANTENNA}

The model of the patch antenna is given in Fig (2). Here $r$ and $\mathrm{R}$ represents the radius of patch and substrate respectively. For a circular polarization the patch antenna must have orthogonal field components with equal amplitude but in phase quadrature. The patch with perturbations can produce circular polarization [12]. Various types of antenna perturbations is available [13]. By in cooperating various perturbations in to the MS, the patch antenna radiate in different polarization. The structure of the unit cell is shown in Fig (3). Here a denotes the length of the unit cell, $\mathrm{b}$ and $\mathrm{c}$ denotes the dimension of the square perturbation, $\mathrm{d}$ denotes the gap between two adjacent unit cells. 


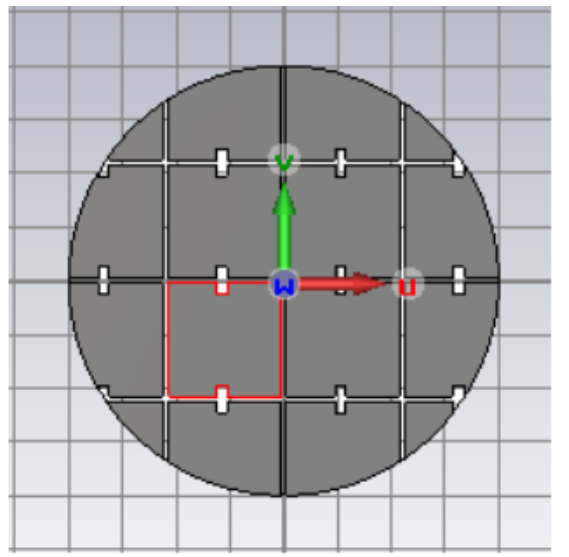

Figure 1 The geometry of MS

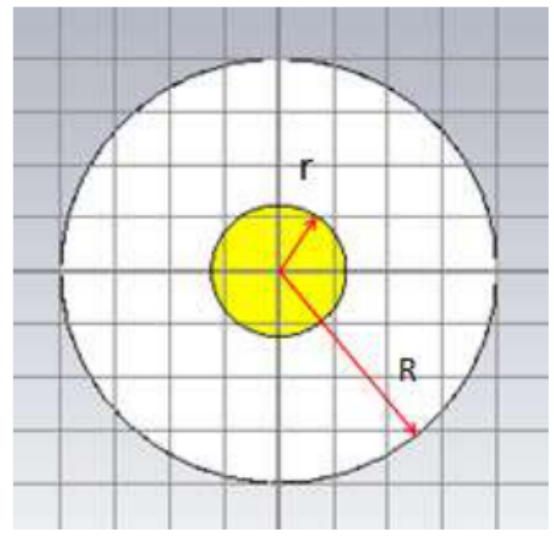

Figure 2 Patch Antenna

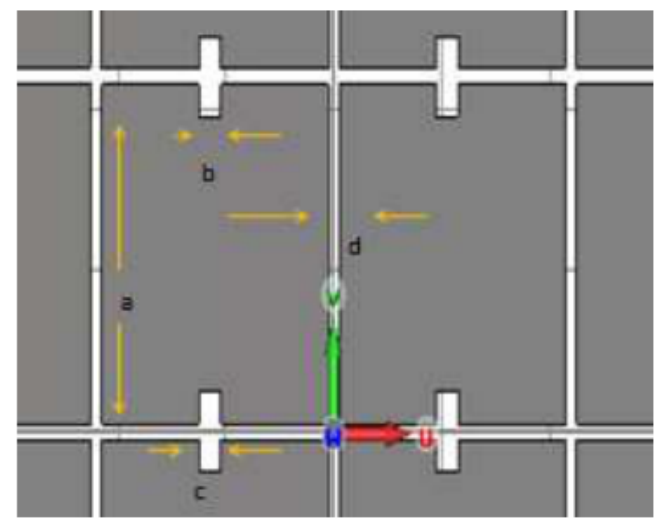

Figure 3 Unit cell

The dimensions of the patch antenna and the metasurface are given in Table 1. These dimensions alter the antenna performance. The radius of the patch (r) antenna is obtained from the equation (1) [15]

Table 1 Dimension of PRMS Antenna (Units: mm)

\begin{tabular}{|c|c|c|c|c|c|}
\hline $\mathrm{a}$ & $\mathrm{b}$ & $\mathrm{c}$ & $\mathrm{d}$ & $\mathrm{r}$ & $\mathrm{R}$ \\
\hline 21 & 2 & 2 & 1 & 16.65 & 40 \\
\hline
\end{tabular}




$$
\mathrm{r}=\frac{F}{\left\{1+\frac{2 h}{\varepsilon_{r} \pi F}\left[\ln \left(\frac{\pi F}{2 h}\right)+1.7726\right]\right\}^{1 / 2}}
$$

$$
\text { Where } \mathrm{F}=\frac{8.791 \times 10^{9}}{f_{r} \sqrt{\varepsilon_{r}}}
$$

Here $f r$ denotes the resonating frequency and $\varepsilon_{r}$ denotes the permittivity of the substrate.

\section{SIMULATION AND RESULTS}

\subsection{Reflection Coefficient (S11)}

The PRMS antenna is designed and simulated. The reflection coefficient at different rotation angle $\theta_{R}$ is studied and it is shown in Fig 4. When $\theta_{R}=0^{\circ}$, the $\mathrm{S} 11$ obtained is $-24 \mathrm{~dB}$ and is shown in Fig 4(a). When $\theta_{R}=45^{\circ}$, the S11 obtainedis- $18 \mathrm{~dB}$ and it is given in Fig 4(b). Here two bands of frequencies are available this is due to the capacitance offered by the MS. When $\theta_{R}=90^{\circ}$, the $\mathrm{S} 11$ obtained is $-21 \mathrm{~dB}$ and it is shown in Fig 4(c). When $\theta_{R}=135^{\circ}$, the S11 obtained is $-21 \mathrm{~dB}$, it is shown in Fig 4(d). Here the antenna is designed at $2.4 \mathrm{GHz}$ because of its wide range of applications. After placing a MS above the antenna there is a frequency shift occur. This is due to the resonance effect of the MS.

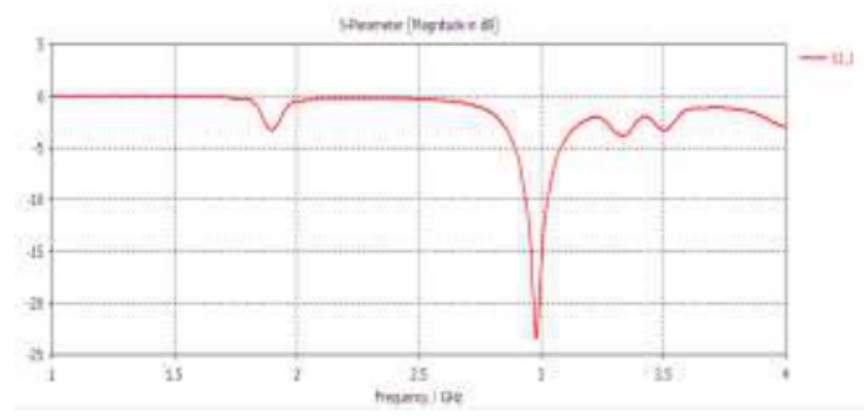

Figure 4 (a): When $\theta_{R}=0^{0}$

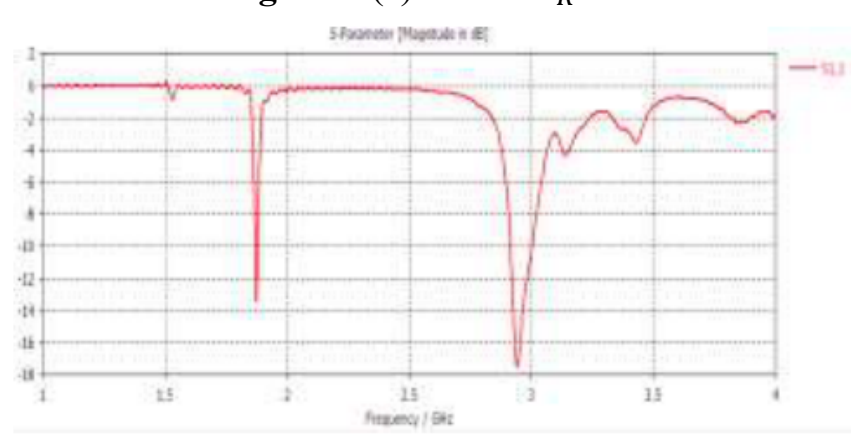

Figure 4 (b): When $\theta_{R}=45^{\circ}$

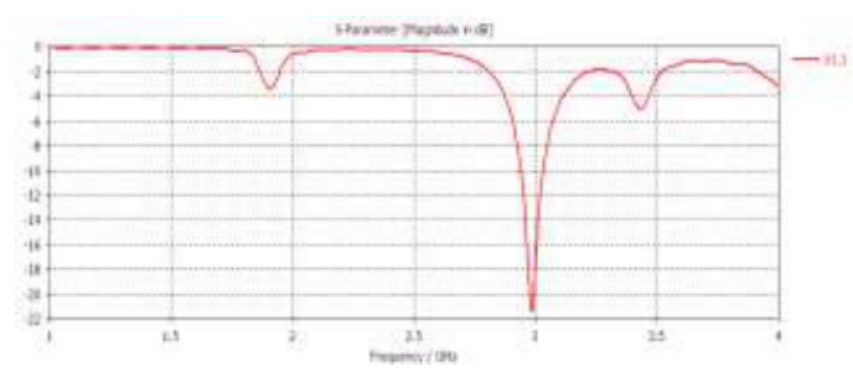

Figure 4 (c): When $\theta_{R}=90^{\circ}$ 


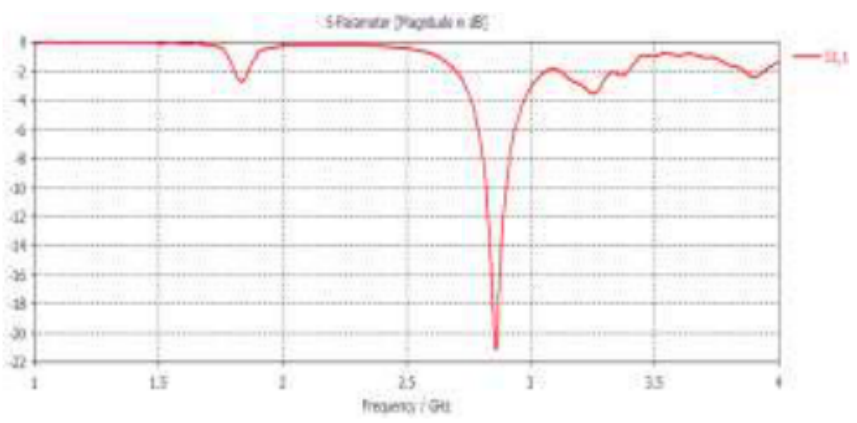

Figure 4(d): When $\theta_{R}=135^{0}$

\subsection{Axial Ratio AR}

The axial ratio is the ratio of orthogonal components of field vectors. The simulated AR of PRMS antenna is shown in below. When the rotation angle $\theta_{R}=0^{0}$ the AR is a high value i.e. $40 \mathrm{~dB}$ shown in Fig 5(a). It indicates that the antenna operated in LP. When $\theta_{R}=45^{\circ}$, the $\mathrm{AR}$ is a small value i.e. $2.46 \mathrm{~dB}$ (which is $<3 \mathrm{~dB}$ ) and it is shown in Fig 5(b). It indicates that the antenna operated in CP. The ARBW obtained is $61.68 \mathrm{MHz}$. The maximum gain of the antenna obtained at $\theta=160^{\circ}$ and $\phi=235^{\circ}$ where $\theta$ and $\phi$ are spherical coordinates. At this specified angles the antenna operates in Right Hand circular polarization (RHCP) and it is shown in Fig 5(c). When $\theta_{R}=90^{\circ}$ the AR obtained is a high value i.e. $31.3 \mathrm{~dB}$ and it is shown in Fig 5(d). It indicates that the antenna operated in LP with an ARBW of 106.1 MHz. When $\theta_{R}=135^{\circ}$, the AR is a small value i.e. $3.26 \mathrm{~dB}$ which is shown in Fig 5(e). There is some cross polarization effect at this angle. So it affects the antenna operations. Here the antenna operates in CP. The maximum gain of the antenna obtained at $\theta=135^{\circ}$ and $\phi=135^{\circ}$. At this specified angles the antenna operates in Left Hand circular polarization (LHCP) and it is shown in Fig 5(f).

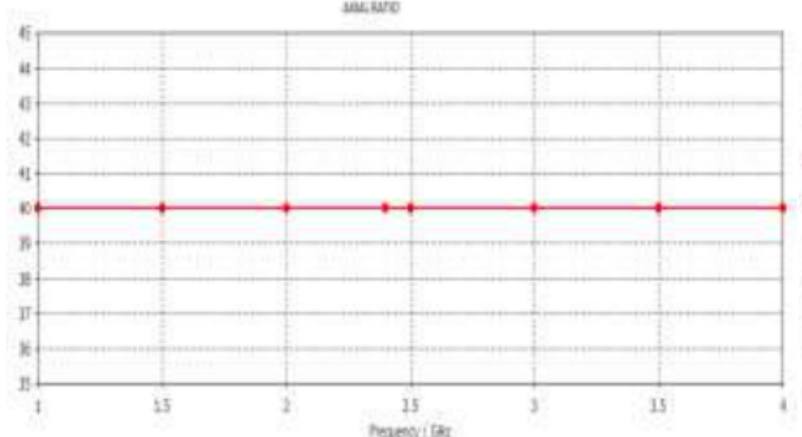

Figure 5 (a): When $\theta_{R}=0^{0}$

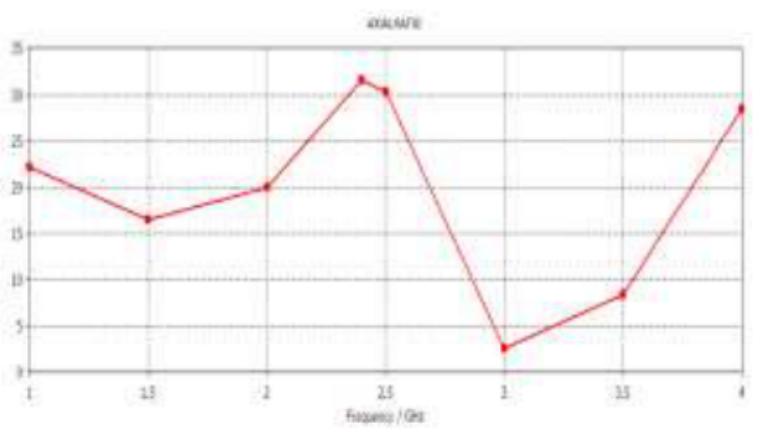

Figure 5 (b): When $\theta_{R}=45^{0}$
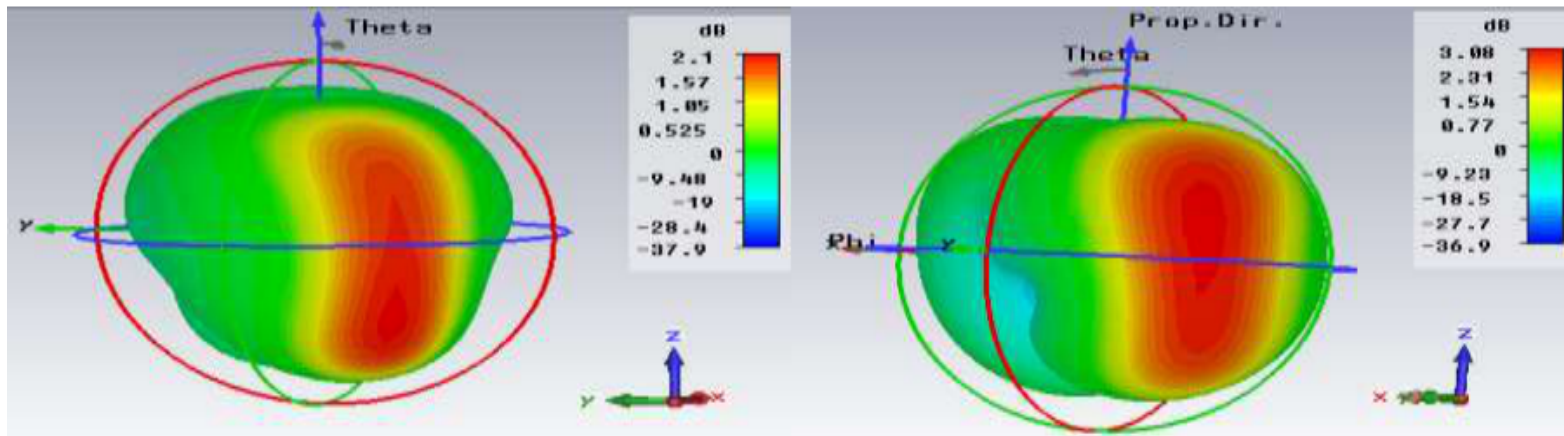

Figure 5(c): Peak gain of RHCP when $\theta_{R}=45^{0}$ Figure 5(f): Peak gain of RHCP when $\theta_{R}=135^{0}$ 
Study on Performance Analysis of Polarization Reconfigurable Antenna Using Metasurface

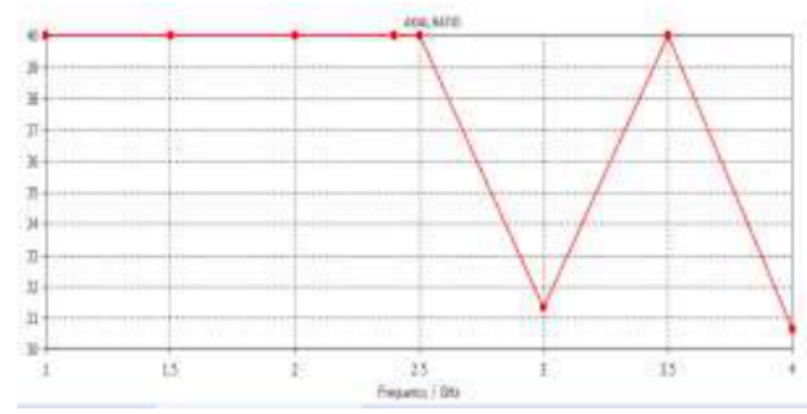

Figure 5.(d): When $\theta_{R}=90^{\circ}$

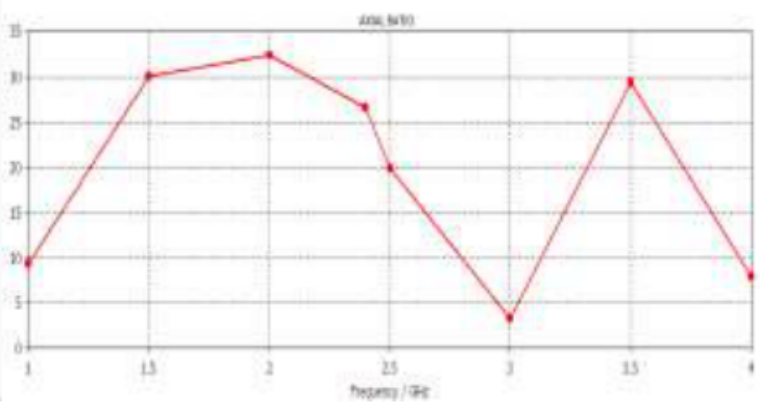

Figure 5(e): When $\theta_{R}=135^{0}$

\subsection{Radiation Pattern}

The radiation pattern is the directional dependence of the strength of radio waves from the antenna or other sources. The simulated radiation pattern of the patch antenna and the PRMS antenna is shown in Fig 6. The patch antenna radiates unidirectional signal. The radiation pattern of a patch antenna is shown in Fig 6(a).

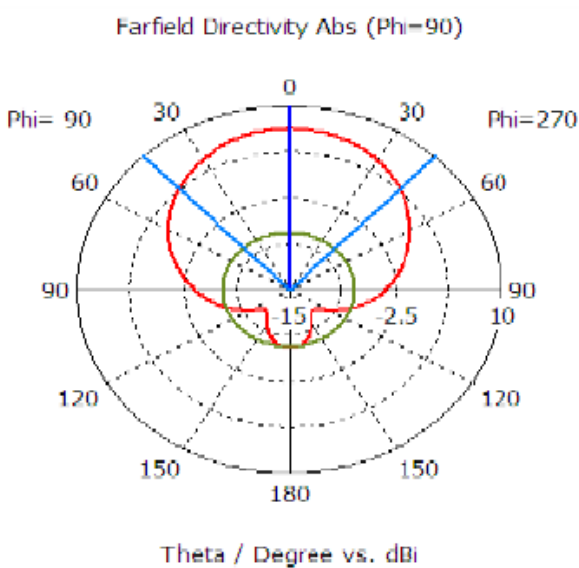

Figure 6 (a): Radiation Pattern of the patch antenna

Farfield Directivity Abs (Phi=90)

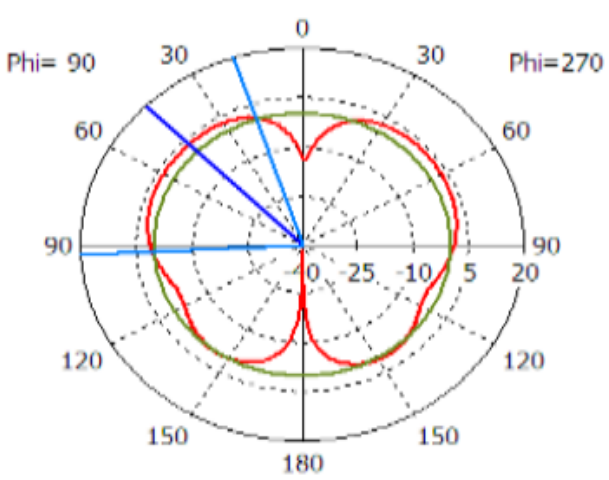

Theta / Degree vs. dBi

Figure 6 (c): Radiation Pattern when $\theta_{R}=45^{\circ}$

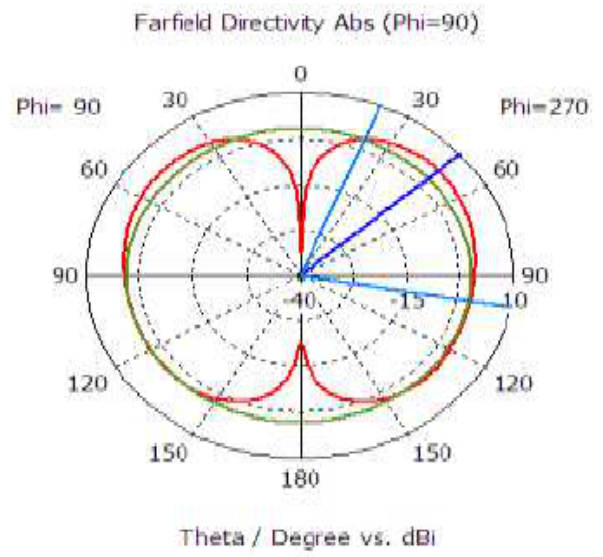

Figure 6 (b): Radiation Pattern when $\theta_{R}=0^{0}$ Farfield Directivity Abs (Phi=90)

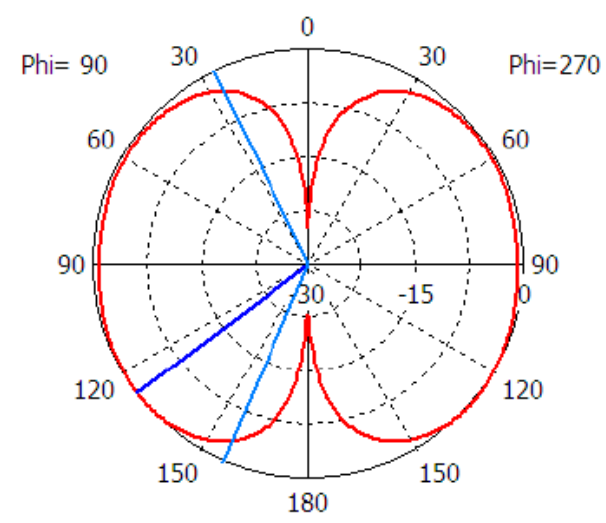

Theta / Degree vs. dBi

Figure 6(d): Radiation Pattern when $\theta_{R}=90^{\circ}$ 


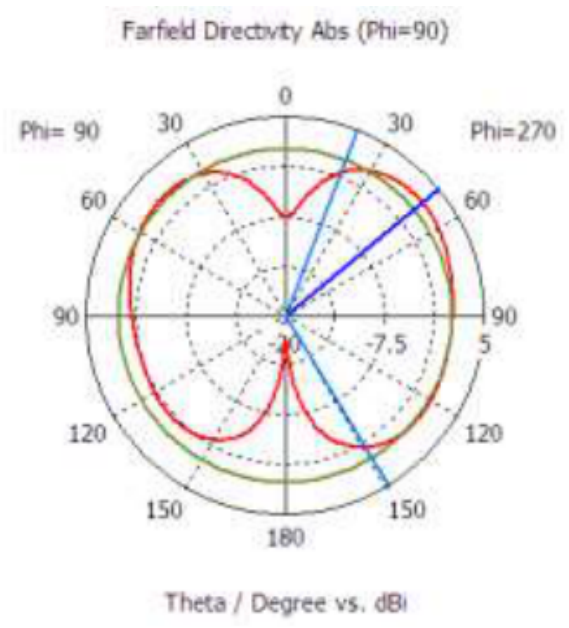

Figure 6 (e): Radiation Pattern when $\theta_{R}=135^{0}$

The main lobe of the radiation pattern is pointed towards $0^{0}$ with a magnitude of $6.91 \mathrm{dBi}$. The $3 \mathrm{~dB}$ angular width is $86.3^{\circ}$ and the side lobe level (SLL) is $-14.1 \mathrm{~dB}$. By placing a metasurface atop the patch antenna, the radiation pattern changed and the directivity reduced. The Fig 6(b) shows the radiation pattern of the antenna when the rotation angle $\theta_{R}=0^{0}$. The main lobe is pointed towards $48^{0}$ with a magnitude of $3.15 \mathrm{dBi}$. The angular width $(3 \mathrm{~dB})$ is $77.7^{\circ}$ and has a side lobe level of $-2.9 \mathrm{~dB}$. Here the directivity of the PRMS antenna reduces due to the presents of side lobes. In Fig 6(c) shows the radiation pattern of the antenna when the rotation angle $\theta_{R}=45^{\circ}$. The main lobe is pointed towards $45^{\circ}$ with a magnitude of 3.53 $\mathrm{dBi}$. The angular width $(3 \mathrm{~dB})$ is $74.7^{\circ}$ and has a side lobe level of $-3 \mathrm{~dB}$. Here the directivity reduces due to the presents of side lobes. In Fig 6(d) shows the radiation pattern of the antenna when the rotation angle $\theta_{R}=90^{\circ}$. The main lobe is pointed towards $127^{\circ}$ with a magnitude of $-.136 \mathrm{dBi}$. The angular width $(3 \mathrm{~dB})$ obtained was $130.5^{0}$.

The main lobe is pointed towards $50^{\circ}$ with a magnitude of $2.22 \mathrm{dBi}$. The angular width (3 $\mathrm{dB}$ ) is $127.5^{\circ}$ with a side lobe level of $-1.2 \mathrm{~dB}$.

\section{CONCLUSIONS}

A PRMS antenna with circular patch antenna and MS was analyzed using the EM simulation Tool CST STUDIO. The polarization of the antenna can be mechanically reconfigured to LP and CP by rotating the MS with respect to the patch antenna. When the MS is at $0^{\circ}$ the PRMS antenna shows a linearly polarized characteristics. By rotating the MS to $45^{\circ}$ the PRMS antenna shows a RHCP characteristics. When the MS is at $90^{\circ}$ the PRMS antenna showed a LP characteristics. When the MS is at $135^{\circ}$ the PRMS antenna showed a LHCP characteristics. The simulated results of polarization reconfigurability, reflection coefficient and the radiation pattern were analyzed. The results showed that the polarization reconfigurability was achieved with in an operating bandwidth of $2.4-3 \mathrm{GHz}$. The directivity of the antenna reduces due to the presence of side lobes. From this analysis using metasurface it can be easily deducted that the simple patch antenna can be made to work with different polarizations by placing the metasurface above the patch antenna. 
Study on Performance Analysis of Polarization Reconfigurable Antenna Using Metasurface

\section{REFERENCES}

[1] H. L. Zhu, S. W. Cheung, X. H. Liu, and T. I. Yuk, "Design of Polarization Reconfigurable Antenna Using Metasurface”, IEEE Transactions On Antennas And Propagation, vol. 62, no. 6, pp 2891-2898 JUNE 2014.

[2] C. Rui-Hung and R. Jeen-Sheen, "Single-Fed Microstrip Patch Antenna with Switchable Polarization," IEEE Trans. Antennas Propag., vol. 56, no. 4, pp. 922 926, Apr. 2008.

[3] R. L. Haupt and M. Lanagan, "Reconfigurable antennas," IEEE Antennas Propag. Mag., vol. 55, no. 1, pp. 49-61, Feb. 2013.

[4] H. T. Friis, C. B. Feldman, and W. M. Sharpless, "The Determination of the Direction of Arrival of Short Radio Waves," Proceedings of the Institute of Radio Engineers, 22, pp. 47-78, Jan 1934.

[5] E. W. Matthews, C. L. Cuccia, and M. D. Rubin, "Technology Considerations for the Use of Multiple Beam Antenna Systems in Communications Satellites," IEEE Transactions on Microwave Theory and Techniques,pp.998-1004, 27, Dec 1979

[6] J. T. Bernhard," Reconfigurable Antennas", San Rafael, CA, Morgan \& Claypool Publishers, 2007.

[7] H. L. Zhu, S. W. Cheung, K. L. Chung, and T. I. Yuk, "Linear-To Circular Polarization Conversion Using Metasurface," IEEE Trans. Antennas Propag., vol. 61, no. 9, pp. 4615-4623, Sep. 2013.

[8] C. L.Holloway, E. F. Kuester, J.A.Gordon, J. O” Hara, J. Booth, and D. R. Smith, "An Overview of The Theory and Applications of Metasurfaces: The twodimensional equivalents of metamaterials," IEEE Antennas Propag. Mag., vol. 54, no. 2, pp. 10-35, Apr. 2012.

[9] Y. J. Sung, T. U. Jang, and Y. S. Kim, “A Reconfigurable Microstrip Antenna for Switchable Polarization," IEEE Microw. Wireless Compon. Lett., vol. 14, no. 11, pp. 534-536, Nov. 2004.

[10] H. L. Zhu, K. L. Chung, X. L. Sun, S. W. Cheung, and T. I. Yuk, "CP Metasurfaced Antennas Excited by LP Sources," in Proc. IEEE Antennas Propag. Soc. Int. Symp. (APSURSI), 2012, pp. 1-2.

[11] K. Boyon, P. Bo, S.Nikolaou,K.Young-Sik, J. Papapolymerou, and M. M. Tentzeris, "A Novel Single-Feed Circular Microstrip Antenna with Reconfigurable Polarization Capability," IEEE Trans. Antennas Propag., vol. 56, no. 3, pp. 630638, Mar. 2008.

[12] Ramesh Garg, Prakash Bhartia, Inder Bahl, Apisak Ittipiboon, "Microstrip Antenna Design Handbook”, Artech House, 2001.

[13] R. Jeen-Sheen and S. Chuang-Jiashih, "Polarization-Diversity Ring Slot Antenna with Frequency Agility," IEEE Trans. Antennas Propag., vol.60, no. 8, pp. 39533957, Aug. 2012.

[14] D. Peroulis, K. Sarabandi, and L. P. B. Katehi, "Design of reconfigurable slot antennas," IEEE Trans. Antennas Propag., vol. 53, no. 2, pp. 645-654, Feb. 2005.

[15] Constantine A Balanis, "Antenna Theory Analysis and Design", Wiley, Third Edition, 2005. 